\title{
Evaluation of Meditation Apps Available on Google Play and Apple Store: An App Review
}

\author{
Raghad MUHIYADDIN ${ }^{\mathrm{a}}$, Alaa ABD-ALRAZAQ ${ }^{\mathrm{a}}$, Zubair SHAH ${ }^{\mathrm{a}}$, Tanvir ALAM $^{\mathrm{a}}$ and \\ Mowafa HOUSEH ${ }^{\mathrm{a}, 1}$ \\ ${ }^{a}$ College of Science and Engineering, Hamad Bin Khalifa University, Doha, Qatar
}

\begin{abstract}
Many meditation apps have been used to improve the mental wellbeing of individuals. However, little information is available regarding the quality of the applications. This study aims to evaluate meditation apps using the Mobile Applications Rating Scale (MARS). A systematic search for meditation apps was performed on both Android Google Play and Apple iOS Store. We used two keywords to search both app stores: meditation and mindfulness. Out of 623 apps identified, 334 apps were excluded due to language, containing only reminders to meditate, or for not being accessible. A total number of 289 apps remained, of which 280 apps were excluded for being information-only focused, containing religious practices, eating habits, exercises, or for not being free. Therefore, nine apps were included in this review for evaluation. The MARS ratings used in this app review were based on scores from a prior study conducted. The mobile app Headspace had the highest average (4), which is rated as 'good' based on MARS. The remaining apps were rated as acceptable with averages that ranged from 3.2-3.7.
\end{abstract}

Keywords. Meditation apps, mindfulness apps, Mobile Applications Ratings Scale (MARS), app review

\section{Introduction}

There is significant research discussing the impact of meditation on an individual's wellbeing. Mindfulness has been found to be beneficial to mental health and is being adopted in medicine both for therapeutic applications as well as in creating protocols for treating conditions such as chronic pain and stress [1]. Meditation is a skill that requires sustained practice and efforts to be effective; this is a challenge for training that implemented in traditional face-to-face or app-based settings. Apps allow for ease of access to practice meditation; additionally, apps designed to interact with users and are aesthetically pleasing are more likely to regularly engage users in performing meditation-based practice activities. Several meditation-based apps have the potential to improve mental wellbeing. Although these apps are easily accessible on various app stores, limited information is available regarding the quality of the applications. The objective of this app review is to evaluate meditation apps available on Android Google Play and Apple iOS Store using the Mobile Applications Rating Scale (MARS) [2].

\footnotetext{
${ }^{1}$ Corresponding Author: Dr. Mowafa Househ, College of Science and Engineering, Hamad Bin Khalifa University, Doha, Qatar; Email: mhouseh@hbku.edu.qa
} 


\section{Methods}

\subsection{App Search and Selection}

A systematic search for meditation apps was conducted in February 2021. For Android, the Google Play Store was used to conduct this search, while the App Store was used for iOS devices. The terms used in searching the app stores were meditation and mindfulness. As for the eligibility criteria, apps were included if they were accessible, included meditation exercises, being written in English, and available for free with in-apppurchases. They were excluded if they only contained reminders for users to meditate, or if they incorporated religious practices, eating habits, exercises, or focused on pregnancy. They were also excluded if they were only information-based and were not for free. In the first phase, after the apps were found, apps were included or excluded based on language and accessibility. In the second phase, the apps were included or excluded based on the app's cost and content.

\subsection{App Evaluation}

The quality of the apps was assessed by using the MARS rating scale. The MARS scale consists of 23 items classified into 3 categories; classification, satisfaction, and quality of the app. The MARS adopts a 5-point scale, with the ratings noted to include 1inadequate, 2-Poor, 3-Acceptable, 4-Good, 5-Excellent. Included apps were rated using the satisfaction category, and four subscales of the app quality category. These include functionality, engagement, information quality, and aesthetics. The MARS score is obtained through computing the mean of the app quality subscales and overall mean score; subjective items are rated separately. MARS ratings used in this review were based on scores from a previously conducted study [3].

\section{Results}

\subsection{Search Results}

By searching Google Play Store and App Store, we identified 246 and 377 apps, respectively. A total of 623 apps were found (Appendix 1). In the preliminary screening phase, we excluded 334 apps for the following reasons: they were not easily accessible $(n=10)$, they were not available in English $(n=38)$, they only reminded users of time to meditate, guided meditation tracks, games, etc. $(n=286)$. In the second phase, we excluded 280 apps because they incorporated religious practices, eating, exercise, or pregnancy $(n=183)$, were information-focused $(n=84)$, or not free $(n=13)$. A total of nine apps were included for evaluation.

\subsection{App Quality Based on MARS}

Appendix 2 shows the rating of the MARS in each category: engagement, functionality, aesthetics, information, satisfaction, and then the overall score of all these categories. Among the apps, Headspace had the highest overall score (4), which indicates a 'good' quality based on MARS. On the other hand, the apps Mindfully Me and Mindfulness 
Coach had the lowest overall score (3.2), which indicates an 'acceptable' quality based on MARS. The overall score ranged between 3.3 and 3.7 for the remaining, indicating an 'acceptable' quality. Engagement and satisfaction were the lowest-rated aspects of the apps with an average of 3.2 whereas functionality was the highest-rated aspect of the apps with an average of 3.2 .

\subsection{Features of Meditation Apps}

Our results show that apps with timers, tracking facilities, the ability to set reminders and link to social media are considered to be high-quality meditation apps [3]. The features we looked for in apps were in-app purchases, timers, reminders, tracking, and social media features (Appendix 3). Only three apps had all the above-mentioned features: Headspace, Mindfulness Daily, and Rhythm Free. On the other hand, one app (i.e., Complete Mindfulness) did not have any of these features. Tracking facilities were the most available feature in the apps $(n=8)$. In-app purchases was the least available feature in the apps $(n=3)$.

\section{Discussion}

\subsection{Principal Findings}

Of the reviewed apps, different methods were used in explaining the concept of meditation and mindfulness. However, there was variance to the extent of explanation, with apps like Headspace and Smiling Mind noted to adopt unique visual methods in explaining the benefits of meditation and mindfulness.

Meditation is a skill that requires sustained practice and efforts for it to be effective, and this is a challenge for both training that is implemented in a traditional face-to-face setting or app-based training. Apps allow for ease of access to meditation practice; apps which have been designed to be interactive and are aesthetically pleasing are most likely to engage users in regularly performing meditation-based practice activities. With regards to engagement, 3.0 was rated to be the minimum acceptable score on the MARS. All of the included apps have met this score. These apps were noted to have high-quality graphics, with interfaces that are simple to adopt and voices that are soothing when guided meditation tasks are being performed. For example, Headspace is noted to use short videos to aid the meditation tracks or serve as a guide during meditation.

Additionally, an app that features a user-base community may motivate users to engage in healthy activities, as these communities could aid users in discussing and sharing their experiences and challenges of meditation practice. This could serve as a support or replacement for the provided support usually present in face-to-face meditation practices. Although more than $60 \%$ of the apps reviewed provided some sort of link to other social media platforms, Headspace was the only app that included community support as part of its features.

\subsection{Practical and Research Implications}

Our review did not evaluate the effectiveness of mediation apps on mental health. We recommend that systematic reviews should be conducted to examine the effectiveness of 
meditation apps on mental health. In addition, we only focused on free or in-app purchase apps, so further app reviews are needed to evaluate the quality of non-free apps. Additionally, we did not search other app stores such as HUAWEI AppGallery. Researchers should consider exploring other app stores in addition to the App Store and Google Play store. Healthcare professionals may be interested in recommending the meditation apps that have the highest overall score and the largest number of features to patients. We also recommend that app developers consider user engagement and satisfaction when creating future apps. Finally, companies may benefit by providing their employees with meditation apps that are free to use (or havein-app purchases), rather than asking workers to use paid apps.

\subsection{Strengths and Limitations}

The study adopted the use of MARS in the measurement of the quality of apps. This was dependent on four objective subscales including information quality, visual aesthetics, functionality and engagement, and one subjective scale of satisfaction. Only the objective scales are incorporated in the total app score as per the quality scales. This indicates the reliability of the measure as to the rating of the quality of apps. It should be noted that MARS scores adopted in this study were based on prior MARS results for the apps identified. We may have excluded several meditations apps because we studied free, English-written apps available at the App Store and Google Play Store.

\section{Conclusions}

Most mediation apps that are available to the public and in the English language have an acceptable quality. However, there is still room to improve mediations apps in order to achieve a higher quality. Healthcare professionals may want to consider recommending mediation apps that have the highest overall score and the largest number of features to their patients. App developers should consider features that would best appeal to user engagement and satisfaction in addition to the remaining aspects of MARS. Systematic reviews should be conducted to evaluate the effectiveness of mediation apps. Appendix files are available at GitHub: https://github.com/rmuhiyaddin/Appendix.git.

\section{References}

[1] Choudhary N, Gill C. Universal Panacea or Organizational Fad? The Role of Context on the Effectiveness of Mindfulness. Academy of Management Proceedings. 2020;2020(1):15355. Crossref, doi:10.5465/ambpp.2020.15355abstract.

[2] Stoyanov SR, Hides L, Kavanagh DJ, Zelenko O, Tjondronegoro D, Mani M. Mobile app rating scale: a new tool for assessing the quality of health mobile apps. JMIR mHealth and uHealth. 2015 Mar 11;3(1):e3422.

[3] Mani M, Kavanagh DJ, Hides L, Stoyanov SR. Review and evaluation of mindfulness-based iPhone apps. JMIR mHealth and uHealth. 2015 Aug 19;3(3):e4328. 